\title{
Numerical Methods for a Model for Compressible Miscible Displacement in Porous Media
}

\author{
By Jim Douglas, Jr. and Jean E. Roberts
}

\begin{abstract}
A nonlinear parabolic system is derived to describe compressible miscible displacement in a porous medium. The system is consistent with the usual model for incompressible miscible displacement. Two finite element procedures are introduced to approximate the concentration of one of the fluids and the pressure of the mixture. The concentration is treated by a Galerkin method in both procedures, while the pressure is treated by either a Galerkin method or by a parabolic mixed finite element method. Optimal order estimates in $L^{2}$ and essentially optimal order estimates in $L^{\infty}$ are derived for the errors in the approximate solutions for both methods.
\end{abstract}

Introduction. We shall consider the single-phase, miscible displacement of one compressible fluid by another in a porous medium under the assumptions that no volume change results from the mixing of the components and that a pressure-density relation exists for each component in a form that is independent of the mixing. These equations of state will imply that the fluids are in the liquid state. Our model will represent a direct generalization of the model [3], [4], [7] that has been treated extensively for incompressible miscible displacement.

The reservoir $\Omega$ will be taken to be of unit thickness and will be identified with a bounded domain in $R^{2}$. We shall omit gravitational terms for simplicity of exposition; no significant mathematical questions arise when the lower order terms are included.

Let $c_{i}$ denote the (volumetric) concentration of the $i$ th component of the fluid mixture, $i=1, \ldots, n$. We assume that a density $\rho_{i}$ can be assigned to the $i$ th component that depends solely on the pressure $p$; moreover, we shall take this equation of state in the form

$$
\frac{d \rho_{i}}{\rho_{i}}=z_{i} d p
$$

where $z_{i}$ is the "constant compressibility" factor $[12, \mathrm{p} .10]$ for the $i$ th component. The assumption of miscibility of the components implies that the Darcy velocity of the fluid is given by

$$
u=-\frac{k}{\mu} \nabla p,
$$

where $k=k(x)$ is the permeability of the rock and $\mu=\mu(c)=\mu\left(c_{1}, \ldots, c_{n}\right)$ is the viscosity of the fluid. Assume that no volume change is induced by mixing the components and that a diffusion coefficient, which can combine the effects of

Received April 14, 1982.

1980 Mathematics Subject Classification. Primary 65N30. 
molecular diffusion and dispersion [13], exists that is component-independent; i.e., let

$$
D=\phi\left\{d_{m} I+|u|\left(d_{l} E(u)+d_{t} E^{\perp}(u)\right)\right\},
$$

where $E(u)=\left[u_{k} u_{l} /|u|^{2}\right]$ is the $2 \times 2$ matrix representing orthogonal projection along the velocity vector and $E^{\perp}(u)=I-E(u)$ its orthogonal complement, and $\phi=\phi(x)$ is the porosity of the rock. Then, conservation of mass of the $i$ th component in the mixture is expressed by the equation

$$
\phi \frac{\partial\left(c_{i} \rho_{i}\right)}{\partial t}+\nabla \cdot\left(c_{i} \rho_{i} u\right)-\nabla \cdot\left(\rho_{i} D \nabla c_{i}\right)=\hat{c}_{i} \rho_{i} q
$$

where $q$ is the external volumetric flow rate, and $\hat{c}_{i}$ is the concentration of the $i$ th component in the external flow; $\hat{c}_{i}$ must be specified at points at which injection (i.e., $q>0)$ takes place, and $\hat{c}_{i}$ is assumed to be equal to $c_{i}$ at production points.

Carry out the differentiation indicated in (1.4), divide by $\rho_{i}$, and use (1.1). The following equation results:

$$
\begin{aligned}
\phi \frac{\partial c_{i}}{\partial t}+\phi z_{i} c_{i} \frac{\partial p}{\partial t}+\nabla \cdot\left(c_{i} u\right)+z_{i} c_{i} u \cdot \nabla p & -\nabla \cdot\left(D \nabla c_{i}\right) \\
& -z_{i} D \nabla c_{i} \cdot \nabla p=\hat{c}_{i} q .
\end{aligned}
$$

If the components are of "slight compressibility" [12, pp. 10-11], then the term $z_{i} c_{i} u \cdot \nabla p$ is effectively quadratic in the velocity, which is small in almost all of the domain, and can be neglected; we shall do so. The term $-z_{i} D \nabla c_{i} \cdot \nabla p=z_{i} \mu k^{-1} u$. $D \nabla c_{i}$ is small in comparison to the term $u \cdot \nabla c_{i}$ that comes from the transport term, as both $z_{i}$ and $D$ are small; thus, we shall neglect this term as well, so that we arrive at the equations

$$
\phi \frac{\partial c_{i}}{\partial t}+\phi z_{i} c_{i} \frac{\partial p}{\partial t}+\nabla \cdot\left(c_{i} u\right)-\nabla \cdot\left(D \nabla c_{i}\right)=\hat{c}_{i} q, \quad i=1, \ldots, n .
$$

It is convenient to transform the system given by (1.6) to obtain an equation for the pressure. Since we assume the fluid to occupy the void space in the rock, it follows that

$$
\sum_{j=1}^{n} c_{j}(x, t)=\sum_{j=1}^{n} \hat{c}_{j}(x, t)=1 .
$$

Add the $n$ equations of (1.6); then,

$$
\phi \sum_{j=1}^{n} z_{j} c_{j} \cdot \frac{\partial p}{\partial t}+\nabla \cdot u=q
$$

or, equivalently,

$$
\phi \sum_{j=1}^{n} z_{j} c_{j} \cdot \frac{\partial p}{\partial t}-\nabla \cdot\left(\frac{k}{\mu} \nabla p\right)=q .
$$

Equation (1.9) can be used along with $n-1$ equations of the form (1.6) to describe the compressible miscible displacement process, or the equations (1.6) can be put into nondivergence form by writing the $\nabla \cdot\left(c_{i} u\right)$ term as $c_{i} \nabla \cdot u+u \cdot \nabla c_{i}$ and 
substituting for $\nabla \cdot u$ by means of (1.8) or (1.9). If this is done, the component conservation equations become

$$
\begin{aligned}
\phi \frac{\partial c_{i}}{\partial t} & +\phi c_{i}\left\{z_{i}-\sum_{j=1}^{n} z_{j} c_{j}\right\} \frac{\partial p}{\partial t} \\
& +u \cdot \nabla c_{i}-\nabla \cdot\left(D \nabla c_{i}\right)=\left(\hat{c}_{i}-c_{i}\right) q, \quad i=1, \ldots, n .
\end{aligned}
$$

The numerical methods that we shall introduce and analyze below can be applied to the $n$ component model; however, for clarity of presentation we shall confine ourselves to a two component displacement problem. Let

$$
\begin{aligned}
c & =c_{1}=1-c_{2}, \\
a(c) & =a(x, c)=k(x) \mu(c)^{-1}, \\
b(c) & =b(x, c)=\phi(x) c_{1}\left\{z_{1}-\sum_{j=1}^{2} z_{j} c_{j}\right\}, \\
d(c) & =d(x, c)=\phi(x) \sum_{j=1}^{2} z_{j} c_{j} .
\end{aligned}
$$

Then, the differential system can be written in the form
(a) $d(c) \frac{\partial p}{\partial t}+\nabla \cdot u=d(c) \frac{\partial p}{\partial t}-\nabla \cdot(a(c) \nabla p)=q$,
(b) $\phi \frac{\partial c}{\partial t}+b(c) \frac{\partial p}{\partial t}+u \cdot \nabla c-\nabla \cdot(D \nabla c)=(\hat{c}-c) q$.

We shall assume that no flow occurs across the boundary:

$$
\begin{array}{ll}
\text { (a) } u \cdot \nu=0 & \text { on } \partial \Omega, \\
\text { (b) } \quad(D \nabla c-c u) \cdot \nu=0 & \text { on } \partial \Omega,
\end{array}
$$

where $\nu$ is the outer normal to $\partial \Omega$. In addition, the initial conditions

(a) $p(x, 0)=p_{0}(x), \quad x \in \Omega$,

(b) $c(x, 0)=c_{0}(x), \quad x \in \Omega$,

must be given.

Note that $b(c)=d(c)=0$ if the compressibilities $z_{1}$ and $z_{2}$ vanish; thus, the model for the compressible problem converges to that of the incompressible model as the fluids tend toward incompressibility.

The purpose of this paper is to formulate and analyze two numerical schemes for approximating the solution of the system (1.12)-(1.14). In both procedures the concentration equation ( $1.12 \mathrm{~b})$ is treated by a parabolic Galerkin procedure. In the first method the pressure equation (1.12a) is treated by a parabolic Galerkin procedure as well, and in the second it is treated by a parabolic mixed finite element technique.

The analysis is given under a number of restrictions. The most important is that the solution is smooth; i.e., $q$ is smoothly distributed, the coefficients are smooth, and the domain has at least the regularity required for a standard elliptic Neumann problem to have $H^{2}(\Omega)$-regularity and more if the piecewise-polynomial spaces used in the finite element procedures have degree greater than one. We shall also consider 
only molecular diffusion, so that $D=\phi(x) d_{m} I$. The coefficients $a, d$, and $\phi$ will be assumed bounded below positively as well as being smooth. Only the continuous-time versions of our methods will be studied here.

The finite element methods will be formulated in Section 2. The scheme based on approximating the pressure by a Galerkin method will be analyzed in Section 3, and the one employing a parabolic mixed finite element method for the pressure will be treated in Section 4. The principal results of the paper are embodied in the $L^{2}$-estimates for the error given in the inequalities (3.35) and (4.31) and in the $L^{\infty}$-estimates (3.38), (3.39), and (4.32). The $L^{2}$-estimates are of optimal order, and, except for a factor of $\log h^{-1}$, so are the $L^{\infty}$-estimates.

2. Formulation of the Finite Element Procedures. Let $h=\left(h_{c}, h_{p}\right)$, where $h_{c}$ and $h_{p}$ are positive. Let $\mathfrak{R}_{h}=\mathfrak{K}_{h_{c}} \subset W^{1, \infty}(\Omega)$ denote a standard finite element space such that

$$
\inf _{z_{h} \in \mathfrak{M}_{h}}\left\|z-z_{h}\right\|_{1, q} \leqslant M\|z\|_{l+1, q} h_{c}^{\prime}
$$

for $z \in W^{l+1, q}(\Omega)$ and $1 \leqslant q \leqslant \infty$, where $\|z\|_{k, q}$ is the norm in the Sobolev space $W^{k, q}(\Omega)$ and $\|z\|_{k}=\|z\|_{k, 2}$. Assume that $\mathfrak{T}_{h}$ is associated with a quasi-regular polygonalization of $\Omega$ and piecewise-polynomial functions of some fixed degree greater than or equal to $l$; thus, all standard inverse relations hold on $\mathfrak{N}_{h}$, and they will be used frequently in the analyses to come. The approximation to the concentration will be denoted by $c_{h}$ and will be given by a map of the time interval $J=[0, T]$ into $\Re_{h}$ based on a standard Galerkin method related to the weak form of $(1.12 b)$ given by

$$
\left(\phi \frac{\partial c}{\partial t}, z\right)+(u \cdot \nabla c, z)+(D \nabla c, \nabla z)+\left(b(c) \frac{\partial p}{\partial t}, z\right)=((\hat{c}-c) q, z)
$$

for $z \in H^{1}(\Omega)$ and $0<t \leqslant T$, where the inner products are to be interpreted to be in $L^{2}(\Omega)$ or $L^{2}(\Omega)^{2}$, as appropriate. If the approximations for the pressure and the Darcy velocity are denoted by $p_{h}$ and $u_{h}$, respectively, then $c_{h}$ is defined to be the solution of the relations

$$
\begin{aligned}
\left(\phi \frac{\partial c_{h}}{\partial t}, z\right)+\left(u_{h} \cdot \nabla c_{h}, z\right) & +\left(D \nabla c_{h}, \nabla z\right) \\
& +\left(b\left(c_{h}\right) \frac{\partial p_{h}}{\partial t}, z\right)=\left(\left(\hat{c}_{h}-c_{h}\right) q, z\right), \quad z \in \Re_{h},
\end{aligned}
$$

for $t \in J$. The function $\hat{c}_{h}$ coincides with $\hat{c}$ where $q>0$ and with $c_{h}$ where $q<0$. Equation (2.3) will be used to give the concentration in connection with both methods for approximating $p$ and $u$. In addition, the initial approximate concentration $c_{h}(0)$ must be determined; several ways to specify $c_{h}(0)$ will be indicated after the requirements on $c(0)-c_{h}(0)$ become clear in the convergence analysis. The boundary condition was used in the derivation of the weak form of the equation.

The Galerkin method for the pressure is based on the weak form of (1.12a) given by

$$
\left(d(c) \frac{\partial p}{\partial t}, v\right)+(a(c) \nabla p, \nabla v)=(q, v), \quad v \in H^{1}(\Omega)
$$


for $0<t \leqslant T$. Let $\Re_{h} \subset W^{1, \infty}(\Omega)$ be a piecewise-polynomial space of degree at least $k$ associated with another quasi-regular polygonalization of $\Omega$; then,

$$
\inf _{v_{h} \in \Re_{h}}\left\|v-v_{h}\right\|_{1, q} \leqslant M\|v\|_{k+1, q} h_{p}^{k}, \quad 1 \leqslant q \leqslant \infty
$$

for $v \in W^{k+1 . q}(\Omega)$. Then, $p_{h}: J \rightarrow \Re_{h}$ will be determined as the solution of

$$
\left(d\left(c_{h}\right) \frac{\partial p_{h}}{\partial t}, v\right)+\left(a\left(c_{h}\right) \nabla p_{h}, \nabla v\right)=(q, v), \quad v \in \Re_{h}, t \in J
$$

starting from initial values $p_{h}(0)$ to be discussed later. The definition of the overall Galerkin procedure is completed by requiring that

$$
u_{h}=-a\left(c_{h}\right) \Gamma p_{h} .
$$

The parabolic mixed method that we shall employ as an alternative way to approximate the pressure is a simple generalization of a method introduced and analyzed by Johnson and Thomé [10]. Let $V=\{v \in H(\operatorname{div} ; \Omega): v \cdot \nu=0$ on $\partial \Omega\}$ and $W=L^{2}(\Omega)$. Then, a saddle-point weak form of $(1.12 \mathrm{a})$ is given by the system

$$
\begin{array}{ll}
\text { (a) }\left(d(c) \frac{\partial p}{\partial t}, w\right)+(\nabla \cdot u, w)=(q, w), & w \in W . \\
\text { (b) }\left(a(c)^{-1} u, v\right)-(\nabla \cdot v, p)=0, & v \in V .
\end{array}
$$

Now, let $V_{h} \times W_{h}$ be a Raviart-Thomas [14], [18] space of index at least $k$ associated with a quasi-regular triangulation or quadrilateralization (or a mixture of the two) of $\Omega$ such that the elements have diameters bounded by $h_{p}$. If $\Omega$ is polygonal, then impose the boundary condition $v \cdot \nu=0$ on $\partial \Omega$ strongly on $V_{h}$. If $\partial \Omega$ is curvilinear and $k=1$, use the boundary element described in Johnson-Thomé [10] and impose the boundary condition on $V_{h}$ by interpolating the condition $v \cdot \nu=0$ at two Gauss points on each boundary edge. (This is a nonconforming feature; the analysis below does not cover this case explicitly, though it should extend without much difficulty to cover it.) Since the outer boundaries of petroleum reservoirs are not known exactly and since the regularity of the solutions of the differential equations is usually quite limited by the presence of sources and sinks, we shall assume $\Omega$ to be polygonal and, for intuitive purposes, think of $k$ as being either zero or one; thus, we shall, in particular, assume in the analysis that the boundary condition is represented exactly in $V_{h}$. The approximation properties for $V_{h} \times W_{h}$ are given by the inequalities

(a) $\inf _{v_{h} \in V_{h}}\left\|v-v_{h}\right\|_{0}=\inf _{v \in V_{h}}\left\|v-v_{h}\right\|_{L^{2}(\Omega)^{2}} \leqslant M\|v\|_{k+1} h_{p}^{k+1}$,

(b) $\inf _{v_{h} \in V_{h}}\left\|\nabla \cdot\left(v-v_{h}\right)\right\|_{0} \leqslant M\left\{\|v\|_{k+1}+\|\nabla \cdot v\|_{k+1}\right\} h_{p}^{k+1}$,

for $v \in V \cap H^{k+1}(\Omega)^{2}$ and, in addition for $(2.9 \mathrm{~b}), \nabla \cdot v \in H^{k+1}(\Omega)$, and

$$
\inf _{w_{h} \in W_{h}}\left\|w-w_{h}\right\|_{0} \leqslant M\|w\|_{k+1} h_{p}^{k+1}, \quad w \in H^{k+1}(\Omega) .
$$


The mixed method for the pressure equation becomes the finding of a map $\left\{u_{h}, p_{h}\right\}$ : $J \rightarrow V_{h} \times W_{h}$ such that

$$
\begin{array}{ll}
\text { (a) }\left(d\left(c_{h}\right) \frac{\partial p_{h}}{\partial t}, w\right)+\left(\nabla \cdot u_{h}, w\right)=(q, w), & w \in W_{h}, \\
\text { (b) }\left(a\left(c_{h}\right)^{-1} u_{h}, v\right)-\left(\nabla \cdot v, p_{h}\right)=0, & v \in V_{h},
\end{array}
$$

for $t \in J$. Initial values must be specified for $p_{h}(0)$; consistent initial values $u_{h}(0)$ can then be computed from $(2.11 \mathrm{~b})$.

The Galerkin procedure defined by the combination of (2.3) and (2.6)-(2.7) represents an essentially traditional finite element approach to the nonlinear parabolic system (1.12), and it is a direct extension of a known method for treating the incompressible miscible problem [3], [4], [7], [8]. Thus, it is of some importance to establish that this, the simplest, finite element method for the compressible problem converges at an asymptotically optimal rate for smooth problems. It is well known that the physical transport dominates the diffusive effects in realistic examples of incompressible miscible displacement. In the liquid-liquid, compressible model studied in this paper, the transport will again dominate the entire process. Thus, it is more important to obtain good approximate velocities than it is to have extreme accuracy in the pressure. As in the incompressible problem [6], this motivates the use of the mixed method (2.11), now of parabolic type, in the calculation of the pressure and the velocity.

The two procedures described above can easily be generalized to treat the $n$-component model. The pressure equation can be handled exactly as above, so long as the argument $c$ in the coefficients $a(c)$ and $d(c)$ is interpreted as the vector $\left\{c_{1}, \ldots, c_{n-1}\right\}$. Instead of a single concentration equation, there will be $n-1$ such equations, and it is convenient to allow the approximation of $c_{i}$ to lie in $\mathfrak{K}_{h, i}$, $i=1, \ldots, n-1$, where these spaces are not required to coincide. In practice, these spaces should be time-dependent [4], and it is possible that the pressure space, either $\Re_{h}$ or $V_{h} \times W_{h}$, should also adapt with time. We shall not treat the time-dependent space procedure here. We believe that our analyses of the two-component model should extend to cover the $n$-component situation.

3. Analysis of the Galerkin Procedure. We have remarked earlier that the location of the boundary of a petroleum reservoir is subject to some uncertainty; moreover, the primary concern in the evaluation of a miscible displacement process will lie in obtaining accurate information about the behavior in the interior of the domain. Consequently, we shall emphasize the interior behavior by considering either the no-flow boundary conditions (1.13) or by assuming $\Omega$ to be a rectangle and by replacing the no-flow boundary conditions by the assumption that the problem is periodic with $\Omega$ as period. This affects the method only in that $\mathfrak{N}_{h}$ and $V_{h}$ should reflect the periodicity.

We shall find it convenient in the analysis to project the solution of the differential problem (1.12) into the finite element spaces by means of coercive elliptic forms associated with the differential system. First, let $\tilde{c}=\tilde{c}_{h}: J \rightarrow \mathfrak{N}_{h}$ be determined by the relations

$$
(D \nabla(c-\tilde{c}), \nabla z)+(u \cdot \nabla(c-\tilde{c}), z)+\lambda(c-\tilde{c}, z)=0, \quad z \in \mathfrak{K}_{h},
$$


for $t \in J$, where the constant $\lambda$ is chosen to be large enough to insure the coercivity of the bilinear form over $H^{1}(\Omega)$.

Similarly, let $\tilde{p}=\tilde{p}_{h}: J \rightarrow \Re_{h}$ satisfy

$$
(a(c) \nabla(p-\tilde{p}), \nabla v)+\mu(p-\tilde{p}, v)=0, \quad v \in \mathscr{K}_{h}, t \in J,
$$

where $\mu$ assures coercivity over $H^{1}(\Omega)$. Let

$$
\begin{array}{ll}
\text { (a) } \zeta=c-\tilde{c}, & \xi=\tilde{c}-c_{h}, \\
\text { (b) } \eta=p-\tilde{p}, & \pi=\tilde{p}-p_{h} .
\end{array}
$$

It is a standard result [2] in the theory of Galerkin methods for elliptic problems that

(a) $\|\zeta\|_{0}+h_{c}\|\zeta\|_{1} \leqslant M\|c\|_{l+1} h_{c}^{l+1}$,

(b) $\|\eta\|_{0}+h_{p}\|\eta\|_{1} \leqslant M\|p\|_{k+1} h_{p}^{k+1}$

for $t \in J$ and a constant that depends on bounds for lower order derivatives of $c$ and p. An argument similar to that used by Wheeler [19] can be applied to the time-differentiated forms of (3.1) and (3.2) to show that

$$
\begin{aligned}
& \text { (a) }\left\|\frac{\partial \zeta}{\partial t}\right\|_{0}+h_{c}\left\|\frac{\partial \zeta}{\partial t}\right\|_{1} \leqslant M\left\{\|c\|_{l+1}+\left\|\frac{\partial c}{\partial t}\right\|_{l+1}\right\} h_{c}^{l+1}, \\
& \text { (b) }\left\|\frac{\partial \eta}{\partial t}\right\|_{0}+h_{p}\left\|\frac{\partial \eta}{\partial t}\right\|_{1} \leqslant M\left\{\|p\|_{k+1}+\left\|\frac{\partial p}{\partial t}\right\|_{k+1}\right\} h_{p}^{k+1},
\end{aligned}
$$

where now $M$ depends on lower order derivatives of $c, p$, and their first derivatives with respect to time.

We shall begin by deriving an evolution inequality for the difference $\pi$ between the projection $\tilde{p}$ and the approximate solution $p_{h}$. The equations (2.6) and (3.2) can be differenced to show that

$$
\begin{aligned}
\left(d\left(c_{h}\right) \frac{\partial \pi}{\partial t}, v\right)+ & \left(a\left(c_{h}\right) \nabla \pi, \nabla v\right) \\
= & \left(\left\{a\left(c_{h}\right)-a(c)\right\} \nabla \tilde{p}, \nabla v\right)+\left(\left\{d\left(c_{h}\right)-d(c)\right\} \frac{\partial \tilde{p}}{\partial t}, v\right) \\
& -\left(d(c) \frac{\partial \eta}{\partial t}, v\right)+\mu(\eta, v), \quad v \in \Re_{h} .
\end{aligned}
$$

Select $\partial \pi / \partial t$ as the test function in (3.6) and observe the relation

$$
\begin{aligned}
& \frac{d}{d t}\left(a\left(c_{h}\right) \nabla \pi, \nabla \pi\right)=2\left(a\left(c_{h}\right) \nabla \pi, \nabla \frac{\partial \pi}{\partial t}\right)+\left(\frac{\partial a}{\partial c}\left(c_{h}\right) \frac{\partial c_{h}}{\partial t} \nabla \pi, \nabla \pi\right) \\
& =2\left(a\left(c_{h}\right) \nabla \pi, \nabla \frac{\partial \pi}{\partial t}\right)-\left(\frac{\partial a}{\partial c}\left(c_{h}\right) \frac{\partial \xi}{\partial t} \nabla \pi, \nabla \pi\right)+\left(\frac{\partial a}{\partial c}\left(c_{h}\right) \frac{\partial \tilde{c}}{\partial t} \nabla \pi, \nabla \pi\right) .
\end{aligned}
$$

Thus,

$$
\begin{aligned}
\left(d\left(c_{h}\right) \frac{\partial \pi}{\partial t},\right. & \left.\frac{\partial \pi}{\partial t}\right)+\frac{1}{2} \frac{d}{d t}\left(a\left(c_{h}\right) \nabla \pi, \nabla \pi\right) \\
= & \frac{1}{2}\left(\frac{\partial a}{\partial c}\left(c_{h}\right) \frac{\partial \xi}{\partial t} \nabla \pi, \nabla \pi\right)-\frac{1}{2}\left(\frac{\partial a}{\partial c}\left(c_{h}\right) \frac{\partial \tilde{c}}{\partial t} \nabla \pi, \nabla \pi\right) \\
& +\left(\left\{a\left(c_{h}\right)-a(c)\right\} \nabla \tilde{p}, \nabla \frac{\partial \pi}{\partial t}\right)+\left(\left\{d\left(c_{h}\right)-d(c)\right\} \frac{\partial \tilde{p}}{\partial t}, \frac{\partial \pi}{\partial t}\right) \\
& -\left(d(c) \frac{\partial \eta}{\partial t}, \frac{\partial \pi}{\partial t}\right)+\mu\left(\eta, \frac{\partial \pi}{\partial t}\right) .
\end{aligned}
$$


The only term in (3.7) that must be handled delicately is that involving $a\left(c_{h}\right)-a(c)$; it will be carried momentarily while the others are treated in a straightforward manner. We have assumed the coefficients $d(c)$ and $a(c)$ to be bounded above and below by positive constants independently of $c$. Thus,

$$
\begin{aligned}
& d_{*}\left\|\frac{\partial \pi}{\partial t}\right\|_{0}^{2}+\frac{1}{2} \frac{d}{d t}\left(a\left(c_{h}\right) \nabla \pi, \nabla \pi\right) \\
& \leqslant K\left[\left\|\frac{\partial \xi}{\partial t}\right\|_{0}\|\nabla \pi\|_{0, x}\|\nabla \pi\|_{0}+\left\{\left\|\frac{\partial \tilde{p}}{\partial t}\right\|_{0, \infty}\left(\|\zeta\|_{0}+\|\xi\|_{0}\right)+\|\eta\|_{0}+\left\|\frac{\partial \eta}{\partial t}\right\|_{0}\right\}\right. \\
& +\frac{1}{2}\left(\left(a\left(c_{h}\right)-a(c)\right) \nabla \tilde{p}, \nabla \frac{\partial \pi}{\partial t}\left\|_{0}+\right\| \frac{\partial \tilde{c}}{\partial t}\left\|_{0, \infty}\right\| \nabla \pi \|_{0}^{2}\right]
\end{aligned}
$$

Make the induction hypothesis that

$$
\|\nabla \pi\|_{0, x} \leqslant K_{1}
$$

for some constant $K_{1}$. Apply (3.4), (3.5), and (3.9) to (3.8) to obtain the inequality

$$
\begin{aligned}
d_{*}\left\|\frac{\partial \pi}{\partial t}\right\|_{0}^{2}+\frac{d}{d t} & \left(a\left(c_{h}\right) \nabla \pi, \nabla \pi\right) \\
\leqslant & K_{2}\left[\|\nabla \pi\|_{0}^{2}+\|\xi\|_{0}^{2}+h_{c}^{2 l+2}+h_{p}^{2 k+2}\right]+\varepsilon\left\|\frac{\partial \xi}{\partial t}\right\|_{0}^{2} \\
& +2\left(\left(a\left(c_{h}\right)-a(c)\right) \nabla \tilde{p}, \nabla \frac{\partial \pi}{\partial t}\right)
\end{aligned}
$$

where $K_{2}$ depends on $K_{1}$ and $\|\partial \tilde{c} / \partial t\|_{0, \infty},\|\partial \tilde{p} / \partial t\|_{0, \infty},\|c\|_{l+1},\|p\|_{k+1}$, and $\|\partial p / \partial t\|_{k+1}$. Now, integrate (3.10) in time to see that

$$
\begin{aligned}
d_{*} \int_{0}^{t}\left\|\frac{\partial \pi}{\partial t}\right\|_{0}^{2} d \tau+ & \left(a\left(c_{h}\right) \nabla \pi, \nabla \pi\right)(t) \\
\leqslant & K_{2}\left[\int_{0}^{t}\left(\|\nabla \pi\|_{0}^{2}+\|\xi\|_{0}^{2}\right) d \tau+\left(h_{c}^{2 l+2}+h_{p}^{2 k+2}\right) t\right] \\
& +\varepsilon \int_{0}^{t}\left\|\frac{\partial \xi}{\partial t}\right\|_{0}^{2} d \tau+2 \int_{0}^{t}\left(\left(a\left(c_{h}\right)-a(c)\right) \nabla \tilde{p}, \nabla \frac{\partial \pi}{\partial t}\right) d \tau \\
& +\left(a\left(c_{h}\right) \nabla \pi, \nabla \pi\right)(0)
\end{aligned}
$$

Let us select an initialization procedure for the pressure and the concentration. Note that the values $p_{h}(0)$ need to be chosen so that $\|\nabla \pi(0)\|_{0}=O\left(h_{p}^{k+1}\right)$ if optimality is to be preserved. While there are many ways to do this, the simplest one from the point of view of our analysis is to take $p_{h}(0)$ to be the elliptic projection of the initial data. Analogous considerations will be seen shortly to apply to the choice of $c_{h}(0)$. Thus, we assign

(a) $p_{h}(0)=\tilde{p}(0) \quad$ or $\pi(0)=0$,

(b) $c_{h}(0)=\tilde{c}(0)$ or $\xi(0)=0$. 
We can avoid an apparent loss of a factor $h_{p}$ by integrating the $\left\{a\left(c_{h}\right)-a(c)\right\}$-term by parts in time. Thus,

$$
\begin{aligned}
\int_{0}^{t}\left(\left\{a\left(c_{h}\right)-a(c)\right\} \nabla \tilde{p}, \nabla \frac{\partial \pi}{\partial t}\right) d \tau & \left(\left\{a\left(c_{h}\right)-a(c)\right\} \nabla \tilde{p}, \nabla \pi\right)(t)-\int_{0}^{t}\left(\left\{a\left(c_{h}\right)-a(c)\right\} \nabla \frac{\partial \tilde{p}}{\partial t}, \nabla \pi\right) d \tau \\
& -\int_{0}^{t}\left(\frac{\partial}{\partial t}\left\{a\left(c_{h}\right)-a(c)\right\} \nabla \tilde{p}, \nabla \pi\right) d \tau \\
\leqslant & K\left[\int _ { 0 } ^ { t } \left\{\left(\|\tilde{p}\|_{1, \infty}+\left\|\frac{\partial \tilde{p}}{\partial t}\right\|_{1, \infty}\right)\left(\|\zeta\|_{0}+\|\xi\|_{0}\right)\right.\right. \\
& \left.+\|\tilde{p}\|_{1, \infty}\left(\left\|\frac{\partial \zeta}{\partial t}\right\|_{0}+\left\|\frac{\partial \xi}{\partial t}\right\|_{0}\right)\right\}\|\nabla \pi\|_{0} d \tau \\
& \left.+\|\tilde{p}(t)\|_{1, \infty}\left(\|\zeta(t)\|_{0}+\|\xi(t)\|_{0}\right)\|\nabla \pi(t)\|_{0}\right] \\
\leqslant & K\left[\int_{0}^{t}\left(\|\nabla \pi\|_{0}^{2}+\|\xi\|_{0}^{2}\right) d \tau+\|\xi(t)\|_{0}^{2}+h_{c}^{2 l+2}\right] \\
& +\varepsilon\|\nabla \pi(t)\|_{0}^{2}+\varepsilon \int_{0}^{t}\left\|\frac{\partial \xi}{\partial t}\right\|_{0}^{2} d \tau,
\end{aligned}
$$

by (3.4). The constant $K$ in (3.13) depends on the $L^{\infty}\left(J ; W^{1, \infty}(\Omega)\right)$-norms of $\tilde{p}$ and $\partial \tilde{p} / \partial t$. Since $k \geqslant 1$, it follows from (3.4), (3.5), the embedding inequality [16]

$$
\|v\|_{0, \infty} \leqslant M\left(\log h_{p}^{-1}\right)^{1 / 2}\|v\|_{1}
$$

holding on $\Re_{h}$ and the trivial inequality $\|v\|_{1, \infty} \leqslant M h^{-1}\|v\|_{1}$ that these norms are bounded for sufficiently smooth solutions. We can now combine (3.11) and (3.13) to obtain our desired evolution inequality for $\pi$ :

$$
\begin{aligned}
& \int_{0}^{t}\left\|\frac{\partial \pi}{\partial t}\right\|^{2} d \tau+\|\nabla \pi(t)\|_{0}^{2} \\
& \quad \leqslant K_{3}\left[\int_{0}^{t}\left(\|\nabla \pi\|_{0}^{2}+\|\xi\|_{0}^{2}\right) d \tau+\|\xi(t)\|_{0}^{2}+h_{c}^{2 l+2}+h_{p}^{2 k+2}\right]+\varepsilon \int_{0}^{t}\left\|\frac{\partial \xi}{\partial t}\right\|_{0}^{2} d \tau
\end{aligned}
$$

where $K_{3}$ depends, in particular, on the induction bound (3.9).

We can turn to the derivation of a corresponding evolution inequality for $\xi$. It follows from (2.2) and (3.1) that

$$
\begin{aligned}
& \left(\phi \frac{\partial \tilde{c}}{\partial t}, z\right)+(u \cdot \nabla \tilde{c}, z)+(D \nabla \tilde{c}, \nabla z)+\left(b(c) \frac{\partial \tilde{p}}{\partial t}, z\right) \\
& =((\hat{c}-c) q, z)-\left(\phi \frac{\partial \zeta}{\partial t}, z\right)+\lambda(\zeta, z)-\left(b(c) \frac{\partial \eta}{\partial t}, z\right), \quad z \in \Re_{h}
\end{aligned}
$$

Note that

$$
(\hat{c}-c)-\left(\hat{c}_{h}-c_{h}\right)= \begin{cases}-(\zeta+\xi), & q>0 \\ 0, & q<0\end{cases}
$$


Difference (3.16) and (2.3) and apply (3.17). Then,

$$
\begin{aligned}
\left(\phi \frac{\partial \xi}{\partial t}, z\right)+\left(u_{h} \cdot \nabla \xi, z\right)+(D \nabla \xi, \nabla z)+\left(b\left(c_{h}\right) \frac{\partial \pi}{\partial t}, z\right) & \\
= & -\left((\zeta+\xi) q^{+}, z\right)-\left(\phi \frac{\partial \zeta}{\partial t}, z\right)+\lambda(\zeta, z)-\left(b(c) \frac{\partial \eta}{\partial t}, z\right) \\
& +\left(\left(u_{h}-u\right) \cdot \nabla \tilde{c}, z\right)+\left(\left(b\left(c_{h}\right)-b(c)\right) \frac{\partial \tilde{p}}{\partial t}, z\right), \quad z \in \Re_{h}
\end{aligned}
$$

Choose the test function $z=\partial \xi / \partial t$, and move the terms $\left(u_{h} \cdot \nabla \xi, z\right)$ and $\left(b\left(c_{h}\right) \partial \pi / \partial t, z\right)$ to the right-hand side. It follows that

$$
\begin{aligned}
\left(\phi \frac{\partial \xi}{\partial t},\right. & \left.\frac{\partial \xi}{\partial t}\right)+\frac{1}{2} \frac{d}{d t}(D \nabla \xi, \nabla \xi) \\
\leqslant & \left.K\left\|\frac{\partial \xi}{\partial t}\right\| \int_{0}\left\|u_{h}\right\|_{0, \infty}\|\nabla \xi\|_{0}+\left\|\frac{\partial \pi}{\partial t}\right\|_{0}+\|\zeta\|_{0}+\|\xi\|_{0}+\left\|\frac{\partial \zeta}{\partial t}\right\|_{0}+\left\|\frac{\partial \eta}{\partial t}\right\|_{0}\right] \\
& +\left(\left(u_{h}-u\right) \cdot \nabla \tilde{c}, \frac{\partial \xi}{\partial t}\right) \\
\leqslant & \varepsilon\left\|\frac{\partial \xi}{\partial t}\right\|_{0}^{2}+K_{4}\left[\|\nabla \xi\|_{0}^{2}+\|\xi\|_{0}^{2}+\left\|\frac{\partial \pi}{\partial t}\right\|_{0}^{2}+h_{c}^{2 l+2}+h_{p}^{2 k+2}\right] \\
& +\left(\left(u_{h}-u\right) \cdot \nabla \tilde{c}, \frac{\partial \xi}{\partial t}\right)
\end{aligned}
$$

where $K_{4}$ depends on $K_{1}$ and various norms of the solution of the differential system. Next, observe that

$$
\begin{aligned}
&\left(\left(u_{h}-u\right) \cdot \nabla \tilde{c}, \frac{\partial \xi}{\partial t}\right)=\left(\left(a(c) \nabla p-a\left(c_{h}\right) \nabla p_{h}\right) \cdot \nabla \tilde{c}, \frac{\partial \xi}{\partial t}\right) \\
&=\left(\left(a(c)-a\left(c_{h}\right)\right) \nabla p_{h} \cdot \nabla \tilde{c}, \frac{\partial \xi}{\partial t}\right) \\
&+\left(a(c) \nabla \pi \cdot \nabla \tilde{c}, \frac{\partial \xi}{\partial t}\right)+\left(a(c) \nabla \eta \cdot \nabla \tilde{c}, \frac{\partial \xi}{\partial t}\right) \\
& \leqslant\left(a(c) \nabla \eta \cdot \nabla \tilde{c}, \frac{\partial \xi}{\partial t}\right)+K_{5}\left[\|\zeta\|_{0}+\|\xi\|_{0}+\|\nabla \pi\|_{0}\right]\left\|\frac{\partial \xi}{\partial t}\right\|_{0},
\end{aligned}
$$

where $K_{5}$ depends on $K_{1}$. If (3.20) is applied to (3.19) and the resulting inequality integrated in time, then it can be seen that

$$
\begin{aligned}
& \int_{0}^{t}\left\|\frac{\partial \xi}{\partial t}\right\|_{0}^{2} d \tau+\|\nabla \xi(t)\|_{0}^{2} \\
& \leqslant K_{6}\left[\int_{0}^{t}\left(\|\xi\|_{1}^{2}+\left\|\frac{\partial \pi}{\partial t}\right\|_{0}^{2}+\|\nabla \pi\|_{0}^{2}\right) d \tau\right. \\
& \left.\quad+h_{c}^{2 l+2}+h_{p}^{2 k+2}+\left|\int_{0}^{t}\left(a(c) \nabla \eta \cdot \nabla \tilde{c}, \frac{\partial \xi}{\partial t}\right) d \tau\right|\right]
\end{aligned}
$$

where again $K_{6}$ depends on $K_{1}$.

We wish to integrate by parts in space in the inner product in the last integral. First, shift $\tilde{c}$ to $c$ :

$$
\left(a(c) \nabla \eta \cdot \nabla \tilde{c}, \frac{\partial \xi}{\partial t}\right)=\left(a(c) \nabla \eta \cdot \nabla c, \frac{\partial \xi}{\partial t}\right)-\left(a(c) \nabla \eta \cdot \nabla \zeta, \frac{\partial \xi}{\partial t}\right)
$$


Now apply the $L^{\infty}(\Omega)$-error estimate [9], [11], [17]

$$
\|\nabla \zeta\|_{0, \infty} \leqslant M\|c\|_{l+1, \infty} h_{c}^{l}
$$

with $M$ depending on some lower order norms of $c$ and $p$, to the last term of (3.22) to see that

$$
\left|\left(a(c) \nabla \eta \cdot \nabla \zeta, \frac{\partial \xi}{\partial t}\right)\right| \leqslant K h_{p}^{k} h_{c}^{\prime}\left\|\frac{\partial \xi}{\partial t}\right\|_{0}
$$

so that

$$
\begin{aligned}
\left|\int_{0}^{t}\left(a(c) \nabla \eta \cdot \nabla \zeta, \frac{\partial \xi}{\partial t}\right) d \tau\right| & \leqslant \varepsilon \int_{0}^{t}\left\|\frac{\partial \xi}{\partial t}\right\|_{0}^{2} d \tau+K h_{p}^{2 k} h_{c}^{2 l} \\
& \leqslant \varepsilon \int_{0}^{t}\left\|\frac{\partial \xi}{\partial t}\right\|_{0}^{2} d \tau+K\left(h_{p}^{2 k+2}+h_{c}^{2 l+2}\right) .
\end{aligned}
$$

Next, if $\langle\cdot, \cdot\rangle$ denotes the inner product on $L^{2}(\partial \Omega)$,

$$
\begin{aligned}
(a(c) & \left.\nabla \eta \cdot \nabla c, \frac{\partial \xi}{\partial t}\right) \\
& =\left\langle\eta, a(c) \frac{\partial c}{\partial \nu} \frac{\partial \xi}{\partial t}\right\rangle-\left(\eta, a(c) \nabla c \cdot \nabla \frac{\partial \xi}{\partial t}+\nabla \cdot(a(c) \nabla c) \frac{\partial \xi}{\partial t}\right),
\end{aligned}
$$

and note that the boundary integral vanishes for either the periodic problem or for the no-flow boundary condition, as $D \nabla c \cdot \nu=0$ on $\partial \Omega$ for either the simple form $D=\phi d_{m} I$ of the diffusion coefficient being analyzed here or the more general form given by (1.3). Since

$$
\left|\int_{0}^{t}\left(\eta, \nabla \cdot(a(c) \nabla c) \frac{\partial \xi}{\partial t}\right) d \tau\right| \leqslant \varepsilon \int_{0}^{t}\left\|\frac{\partial \xi}{\partial t}\right\|_{0}^{2} d \tau+K h_{p}^{2 k+2},
$$

we are left with the single term involving $\nabla \partial \xi / \partial t$. This term should be integrated by parts in time:

$$
\begin{aligned}
\int_{0}^{t}(\eta, a(c) & \left.\nabla c \cdot \nabla \frac{\partial \xi}{\partial t}\right) d \tau \\
= & (\eta, a(c) \nabla c \cdot \nabla \xi)(t) \\
& -\int_{0}^{t}\left[\left(\frac{\partial \eta}{\partial t}, a(c) \nabla c \cdot \nabla \xi\right)+\left(\eta \frac{\partial}{\partial t}(a(c) \nabla c), \nabla \xi\right)\right] d \tau \\
\leqslant & \varepsilon\|\nabla \xi(t)\|_{0}^{2}+K\left[\int_{0}^{t}\|\nabla \xi\|_{0}^{2} d \tau+h_{p}^{2 k+2}\right]
\end{aligned}
$$

The various relations (3.22)-(3.27) and the trivial inequality

$$
\|\xi(t)\|_{0}^{2}=\int_{0}^{t} \frac{d}{d t}\|\xi\|_{0}^{2} d \tau \leqslant \varepsilon \int_{0}^{t}\left\|\frac{\partial \xi}{\partial t}\right\|_{0}^{2} d \tau+K \int_{0}^{t}\|\xi\|_{0}^{2} d \tau
$$

can be combined with (3.21) to show that

$$
\begin{aligned}
\int_{0}^{t}\left\|\frac{\partial \xi}{\partial t}\right\|_{0}^{2} d \tau & +\|\xi(t)\|_{1}^{2} \\
& \leqslant K_{7}\left[\int_{0}^{t}\left(\|\xi\|_{1}^{2}+\left\|\frac{\partial \pi}{\partial t}\right\|_{0}^{2}+\|\nabla \pi\|_{0}^{2}\right) d \tau+h_{p}^{2 k+2}+h_{c}^{2 l+2}\right]
\end{aligned}
$$

with $K_{7}$ dependent on $K_{1}$. 
The object is to combine (3.15) and (3.29) in a judicious manner so that we can obtain estimates in $L^{\infty}\left(J ; H^{1}(\Omega)\right)$ for both $\pi$ and $\xi$, along with estimates in $L^{2}\left(J ; L^{2}(\Omega)\right)$ for $\partial \pi / \partial t$ and $\partial \xi / \partial t$. First, use the analogue of (3.28) for $\pi$ to replace the $L^{2}(\Omega)^{2}$-norms of $\nabla \pi$ by $H^{1}(\Omega)$-norms of $\pi$, and simultaneously apply (3.28) itself on the right-hand side of (3.15). Thus,

$$
\begin{aligned}
\int_{0}^{t}\left\|\frac{\partial \pi}{\partial t}\right\|_{0}^{2} d \tau+\|\pi(t)\|_{1}^{2} \leqslant & K_{8}\left[\int_{0}^{t}\left(\|\pi\|_{1}^{2}+\|\xi\|_{0}^{2}\right) d \tau+h_{p}^{2 k+2}+h_{c}^{2 l+2}\right] \\
& +\varepsilon \int_{0}^{t}\left\|\frac{\partial \xi}{\partial t}\right\|^{2} d \tau
\end{aligned}
$$

where, as usual, the constant $K_{8}$ depends on $K_{1}$. Now, take a $\left(K_{7}+1\right)$-multiple of (3.30) and add it to (3.29). Require that the $\varepsilon$ appearing in (3.30) be sufficiently small that $\left(K_{7}+1\right) \varepsilon \leqslant \frac{1}{2}$; this increases $K_{8}$, but not $K_{7}$. After a minor amount of collecting terms, we see that

$$
\begin{aligned}
\int_{0}^{t}\left(\left\|\frac{\partial \xi}{\partial t}\right\|_{0}^{2}+\left\|\frac{\partial \pi}{\partial t}\right\|_{0}^{2}\right) d \tau+ & \|\xi(t)\|_{1}^{2}+\|\pi(t)\|_{1}^{2} \\
& \leqslant K_{9}\left[\int_{0}^{t}\left(\|\xi\|_{1}^{2}+\|\pi\|_{1}^{2}\right) d \tau+h_{p}^{2 k+2}+h_{c}^{2 l+2}\right],
\end{aligned}
$$

with $K_{9}$ depending on $K_{1}$. Thus, it follows from the Gronwall lemma that

$$
\begin{aligned}
&\|\xi\|_{L^{x}\left(J ; H^{1}(\Omega)\right)}+\left\|\frac{\partial \xi}{\partial t}\right\|_{L^{2}\left(J ; L^{2}(\Omega)\right)}+\|\pi\|_{L^{x}\left(J ; H^{1}(\Omega)\right)}+\left\|\frac{\partial \pi}{\partial t}\right\|_{L^{2}\left(J: L^{2}(\Omega)\right)} \\
& \leqslant K^{*}\left(h_{p}^{k+1}+h_{c}^{l+1}\right),
\end{aligned}
$$

where $K^{*}$ is supposed to depend on the induction bound $K_{1}$ for $\nabla \pi$ in $L^{x}(\Omega)$. The quasi-regularity of the polygonalization for the space $\Re_{h}$ implies that

$$
\|\nabla \pi\|_{L^{x}\left(J ; L^{x}(\Omega)\right)} \leqslant K^{\prime}\left(h_{p}^{k}+h_{c}^{l+1} h_{p}^{-1}\right) .
$$

Adopt the restriction between the two spaces that

$$
h_{c}^{l+1} h_{p}^{-1} \rightarrow 0 \text { as } h \rightarrow 0 .
$$

If (3.34) holds, then $K_{1}$ could have been taken to the arbitrarily small and a choice can be made for $K^{*}$ that is independent of $K_{1}$.

A justification of the induction hypothesis can be given at this point. What has been shown is that, if $\nabla \pi$ is bounded uniformly by some constant $K_{1}$, then $\nabla \pi$ tends uniformly to zero as $h_{p}$ and $h_{c}$ tend to zero subject to (3.34). Now, the initial conditions were chosen so that $\nabla \pi(0)=0$; hence, for any fixed pair $\left(h_{p}, h_{c}\right),(3.9)$ holds for $0 \leqslant t \leqslant T_{h}$ for some $T_{h}>0$. The implication of (3.3) and (3.4) is that $T_{h}=T$ for $\left(h_{p}, h_{c}\right)$ sufficiently small; i.e., (3.9) holds for small $\left(h_{p}, h_{c}\right)$.

The relation (3.34) is unimportant, since normally the parameters $h_{p}$ and $h_{c}$ would be selected such that

$$
0<M_{1} \leqslant h_{c}^{l+1} h_{p}^{-(k+1)} \leqslant M_{2}
$$

so that

$$
h_{c}^{l+1} h_{p}^{-1} \leqslant M h_{c}^{(l+1) k /(k+1)} \rightarrow 0 \quad \text { as } h \rightarrow 0
$$


Error estimates in $L^{2}(\Omega)$ result from combining (3.32) with the bounds given by (3.4) and (3.5) for the projection errors. We see that, under the constraint (3.34),

$$
\begin{aligned}
& \left\|c-c_{h}\right\|_{L^{x}\left(J: L^{2}(\Omega)\right)}+h_{c}\left\|c-c_{h}\right\|_{L^{x}\left(J: H^{1}(\Omega)\right)}+\left\|\frac{\partial c}{\partial t}-\frac{\partial c_{h}}{\partial t}\right\|_{L^{2}\left(J: L^{2}(\Omega)\right)} \\
& \quad+\left\|p-p_{h}\right\|_{L^{x}\left(J: I^{2}(\Omega)\right)}+h_{p}\left\|u-u_{h}\right\|_{\left.L^{x}\left(J: I^{2}(\Omega)\right)^{2}\right)}+\left\|\frac{\partial p}{\partial t}-\frac{\partial p_{h}}{\partial t}\right\|_{L^{2}\left(J: I^{2}(\Omega)\right)} \\
& \leqslant K\left(h_{p}^{h+1}+h_{c}^{l+1}\right) .
\end{aligned}
$$

where the constant $K$ depends on spatial derivatives of order not greater than $l+1$ of $c$ and $\partial c / \partial t$ and of order not greater than $k+1$ of $p$ and $\partial p / \partial t$. The use of the $L^{\infty}$-estimate (3.23) means that $K$ involves a bit more than the minimal regularity of $c$ and $p$ that would be required by the approximation properties of the spaces $\Omega_{h}$ and $\Re_{h}$ in order that a bound of the form derived could hold; however, our primary objective of obtaining optimal rates of convergence has been achieved.

We can derive an almost optimal $L^{\infty}$-estimate from (3.32), as well. The bound (3.23) can be expanded to the more complete form [9], [11], [17]
(a) $\|\zeta\|_{0, \infty}+h_{c}\|\zeta\|_{1, \infty} \leqslant M\|c\|_{l+1, \infty} h_{c}^{1+1}\left(\log h_{c}^{-1}\right)^{\alpha}$,
(b) $\|\eta\|_{0, \infty}+h_{p}\|\eta\|_{1, \infty} \leqslant M\|p\|_{l+1, \infty} h_{p}^{k+1}\left(\log h_{p}^{-1}\right)^{\beta}$.

where $\alpha=1$ for $l=1, \alpha=0$ for $l>1, \beta=1$ for $k=1$, and $\beta=0$ for $k>1$. The embedding inequality (3.14) and the bound (3.32) imply that

$$
\begin{aligned}
\|\xi\|_{L^{x}\left(J: L^{\infty}(\Omega)\right)} & +\|\pi\|_{L^{x}\left(J: L^{x}(\Omega)\right)} \\
& \leqslant K\left(h_{p}^{k+1}+h_{c}^{l+1}\right)\left[\left(\log h_{p}^{-1}\right)^{1 / 2}+\left(\log h_{c}^{-1}\right)^{1 / 2}\right] .
\end{aligned}
$$

Thus, it follows for $k \geqslant 1$ and $l \geqslant 1$ that

$$
\begin{aligned}
\left\|c-c_{h}\right\|_{L^{\infty}\left(J: L^{\infty}(\Omega)\right)} & +\left\|p-p_{h}\right\|_{L^{\infty}\left(J: L^{\infty}(\Omega)\right)} \\
& \leqslant K\left(h_{p}^{k+1}+h_{c}^{l+1}\right)\left(\log h_{p}^{-1}+\log h_{c}^{-1}\right) .
\end{aligned}
$$

The bounds (3.23) and (3.33) combine to give the estimate

$$
\left\|u-u_{h}\right\|_{L^{\infty}\left(J: L^{\infty}(\Omega)\right)} \leqslant K\left(h_{p}^{k}+h_{c}^{l+1} h_{p}^{-1}\right) ;
$$

if $h_{p}^{k+1}$ and $h_{c}^{l+1}$ are of the same size, then this estimate is of optimal order. Both (3.38) and (3.39) assume (3.34) to hold.

We have shown that the standard Galerkin procedure as applied to the parabolic system describing compressible miscible displacement is asymptotically of optimal order in $L^{2}(\Omega)$ and almost so in $L^{\infty}(\Omega)$ when the solution of the problem is smooth. Actual petroleum reservoir simulation would lead to less smoothness than we have assumed, and the argument that we have presented does not cover the case of sources and sinks, the wells that occur in a petroleum reservoir. Two arguments [6], [8], [12] have been constructed for the incompressible problem to handle sources and sinks, but each involves the equally unphysical assumption that the viscosity is independent of the concentration. 
4. Analysis of the Mixed Method Procedure. We again find the projection of the solution of the differential problem into the finite element space $\mathfrak{T}_{h} \times V_{h} \times W_{h}$ valuable in the analysis of the convergence of the method resulting from the combination of $(2.3)$ and $(2.11)$. Let $\tilde{c}$, the projection of the concentration, remain defined by (3.1), and let $\{\tilde{u}, \tilde{p}\}$, the projection of the Darcy velocity and the pressure, be given as the solution of the elliptic mixed method equations

$$
\begin{array}{ll}
\text { (a) }\left(d(c) \frac{\partial p}{\partial t}, w\right)+(\nabla \cdot \tilde{u}, w)=(q, w), & w \in W_{h}, \\
\text { (b) }(\alpha(c) \tilde{u}, v)-(\nabla \cdot v, \tilde{p})=0, & v \in V_{h}, \\
\text { (c) }(\tilde{p}, 1)=(p, 1), &
\end{array}
$$

where $\alpha(c)=a(c)^{-1}$ and $t \in J$. Set

$$
\begin{array}{ll}
\text { (a) } \eta=p-\tilde{p}, & \pi=\tilde{p}-p_{h}, \\
\text { (b) } \rho=u-\tilde{u}, & \sigma=\tilde{u}-u_{h},
\end{array}
$$

and retain the definitions $\zeta=c-\tilde{c}$ and $\xi=\tilde{c}-c_{h}$.

Initialize the approximations to concentration and pressure by taking

$$
\begin{aligned}
& \text { (a) } c_{h}(0)=\tilde{c}(0) \quad \text { or } \quad \xi(0)=0 \\
& \text { (b) } p_{h}(0)=\tilde{p}(0) \quad \text { or } \quad \pi(0)=0 .
\end{aligned}
$$

It follows that $\sigma(0)=0$. Note that $\tilde{u}(0)$ and $\tilde{p}(0)$ can be computed, since $\partial p / \partial t(0)$ can be evaluated using the differential system.

If the relations (4.1) and (2.8) are differenced, we see that the projection error satisfies the equations

$$
\begin{array}{ll}
\text { (a) }(\nabla \cdot \rho, w)=0, & w \in W_{h}, \\
\text { (b) } \quad(\alpha(c) \rho, v)-(\nabla \cdot v, \eta)=0, & v \in V_{h} .
\end{array}
$$

A projection almost identical to this one was analyzed in [6]; there, the average value of $\tilde{p}$ over $\Omega$ was set to zero to coincide with the average value of $p$, and here (4.1c) imposes the equality of the average values of $\tilde{p}$ and $p$. The effect of adding (4.1c) is to drop the necessity that the "inf sup" condition $\mathrm{H} 2$ of Brezzi [1] hold for the constant functions in $W_{h}$. Thus, the estimate [6, (5.2)] given by

$$
\|\rho\|_{H(\operatorname{div} ; \Omega)}+\|\eta\|_{0} \leqslant M\|p\|_{k+3} h_{p}^{k+1}
$$

holds for $t \in J$ with the constant $M$ dependent only on bounds for the coefficient $\alpha(c)$, but not on $c$ itself.

We shall need bounds on $\partial \rho / \partial t$ and $\partial \eta / \partial t$ as well. Differentiate the error equations (4.4) with respect to $t$ to obtain the relations

$$
\begin{array}{ll}
\text { (a) }\left(\nabla \cdot \frac{\partial \rho}{\partial t}, w\right)=0, & w \in W_{h} \\
\text { (b) }\left(\alpha(c) \frac{\partial \rho}{\partial t}, v\right)-\left(\nabla \cdot v, \frac{\partial \eta}{\partial t}\right)=-\left(\frac{\partial \alpha}{\partial c}(c) \frac{\partial c}{\partial t} \rho, v\right), & v \in V_{h}
\end{array}
$$


Let $q \in W_{h}$ and $s \in V_{h}$, and write $\partial \eta / \partial t$ and $\partial \rho / \partial t$ in the forms $\partial \eta / \partial t=(q-$ $\partial \tilde{p} / \partial t)+(\partial p / \partial t-q)$ and $\partial \rho / \partial t=(s-\partial \tilde{u} / \partial t)+(\partial u / \partial t-s)$. Assume that $(q, 1)$ $=(p, 1)$. Then,

$$
\begin{aligned}
& \text { (a) }\left(\nabla \cdot\left(s-\frac{\partial \tilde{u}}{\partial t}\right), w\right)=\left(\nabla \cdot\left(s-\frac{\partial u}{\partial t}\right), w\right), \quad w \in W_{h}, \\
& \text { (b) }\left(\alpha\left(s-\frac{\partial \tilde{u}}{\partial t}\right), v\right)-\left(\nabla \cdot v, q-\frac{\partial \tilde{p}}{\partial t}\right) \\
& =\left(\frac{\partial \alpha}{\partial c} \frac{\partial c}{\partial t} \rho, v\right)+\left(\alpha\left(s-\frac{\partial u}{\partial t}\right), v\right)-\left(\nabla \cdot v, q-\frac{\partial p}{\partial t}\right), \quad v \in V_{h} .
\end{aligned}
$$

The boundedness of the inverse operator for the mixed method over $V_{h} \times W_{h}$ demonstrated in $[6,(4.4)]$ implies that

$$
\left\|s-\frac{\partial \tilde{u}}{\partial t}\right\|_{H(\operatorname{div} ; \Omega)}+\left\|q-\frac{\partial \tilde{p}}{\partial t}\right\|_{0} \leqslant M\left\{\|\rho\|_{0}+\left\|s-\frac{\partial u}{\partial t}\right\|_{H(\operatorname{div} ; \Omega)}+\left\|q-\frac{\partial p}{\partial t}\right\|_{0}\right\} .
$$

Thus,

$$
\begin{aligned}
\left\|\frac{\partial \rho}{\partial t}\right\|_{H(\operatorname{div} ; \Omega)}+ & \left\|\frac{\partial \eta}{\partial t}\right\|_{0} \\
& \leqslant(M+1)\left\{\|\rho\|_{0}+\inf _{s \in V_{h}}\left\|\frac{\partial u}{\partial t}-s\right\|_{H(\operatorname{div} ; \Omega)}+\inf _{q \in W_{h}}\left\|\frac{\partial p}{\partial t}-q\right\|_{0}\right\} \\
& \leqslant M^{\prime}\left\{\|p\|_{k+3}+\left\|\frac{\partial p}{\partial t}\right\|_{k+3}\right\} h_{p}^{k+1} .
\end{aligned}
$$

Now, difference (4.1a) and (2.11a) and take $\partial \pi / \partial t$ as the test function:

$$
\begin{aligned}
\left(d\left(c_{h}\right) \frac{\partial \pi}{\partial t}, \frac{\partial \pi}{\partial t}\right) & \left(\nabla \cdot \sigma, \frac{\partial \pi}{\partial t}\right) \\
& =\left(\left(d\left(c_{h}\right)-d(c)\right) \frac{\partial \tilde{p}}{\partial t}, \frac{\partial \pi}{\partial t}\right)-\left(d(c) \frac{\partial \eta}{\partial t}, \frac{\partial \pi}{\partial t}\right) .
\end{aligned}
$$

Next, difference (4.1b) and (2.11b), differentiate with respect to time, and then choose $\sigma$ for the test function; the following equation results:

$$
\left(\frac{\partial}{\partial t}\left(\alpha\left(c_{h}\right) \sigma\right), \sigma\right)-\left(\nabla \cdot \sigma, \frac{\partial \pi}{\partial t}\right)=\left(\frac{\partial}{\partial t}\left[\left(\alpha\left(c_{h}\right)-\alpha(c)\right) \tilde{u}\right], \sigma\right) .
$$

Since

$$
\frac{d}{d t}\left(\alpha\left(c_{h}\right) \sigma, \sigma\right)=2\left(\frac{\partial}{\partial t}\left(\alpha\left(c_{h}\right) \sigma\right), \sigma\right)-\left(\frac{\partial \alpha}{\partial c}\left(c_{h}\right) \frac{\partial c_{h}}{\partial t} \sigma, \sigma\right)
$$

we see that

$$
\text { (4.12) } \begin{aligned}
\frac{1}{2} \frac{d}{d t}\left(\alpha\left(c_{h}\right) \sigma, \sigma\right)-\left(\nabla \cdot \sigma, \frac{\partial \pi}{\partial t}\right) \\
=\left(\frac{\partial}{\partial t}\left[\left(\alpha\left(c_{h}\right)-\alpha(c)\right) \tilde{u}\right], \sigma\right)-\frac{1}{2}\left(\frac{\partial \alpha}{\partial c}\left(c_{h}\right) \frac{\partial c_{h}}{\partial t} \sigma, \sigma\right) .
\end{aligned}
$$


Momentarily, we shall add (4.10) and (4.12); however, let us bound the four terms on the right-hand sides of the two relations. Note that (4.5), (4.9), and quasi-regularity imply that $\partial \tilde{p} / \partial t, \tilde{u}$, and $\partial \tilde{u} / \partial t$ are bounded in $L^{\infty}(\Omega)$; we have seen previously that $\partial \tilde{c} / \partial t$ is bounded. First

$$
\begin{aligned}
\left|\left(\left(d\left(c_{h}\right)-d(c)\right) \frac{\partial \tilde{p}}{\partial t}, \frac{\partial \pi}{\partial t}\right)\right| & \leqslant K\left\{\|\xi\|_{0}+\|\zeta\|_{0}\right\}\left\|\frac{\partial \pi}{\partial t}\right\|_{0} \\
& \leqslant \varepsilon\left\|\frac{\partial \pi}{\partial t}\right\|_{0}^{2}+K\left[\|\xi\|_{0}^{2}+h_{c}^{2 l+2}\right] .
\end{aligned}
$$

Then, from (4.9),

$$
\left|\left(d(c) \frac{\partial \eta}{\partial t}, \frac{\partial \pi}{\partial t}\right)\right| \leqslant \varepsilon\left\|\frac{\partial \pi}{\partial t}\right\|_{0}^{2}+K h_{p}^{2 k+2} .
$$

Next,

$$
\begin{aligned}
\mid\left(\frac { \partial } { \partial t } \left[\left(\alpha\left(c_{h}\right)\right.\right.\right. & -\alpha(c)) \tilde{u}], \sigma) \mid \\
& =\left|\left(\tilde{u}\left[\frac{\partial \alpha}{\partial c}\left(c_{h}\right) \frac{\partial c_{h}}{\partial t}-\frac{\partial \alpha}{\partial c}(c) \frac{\partial c}{\partial t}\right]+\left[\alpha\left(c_{h}\right)-\alpha(c)\right] \frac{\partial \tilde{u}}{\partial t}, \sigma\right)\right| \\
& \leqslant K\|\sigma\|_{0}\left\{\left\|\frac{\partial \xi}{\partial t}\right\|_{0}+\left\|\frac{\partial \zeta}{\partial t}\right\|_{0}+\|\xi\|_{0}+\|\zeta\|_{0}\right\} \\
& \leqslant \varepsilon\left\|\frac{\partial \xi}{\partial t}\right\|_{0}^{2}+K\left\{\|\xi\|_{0}^{2}+\|\sigma\|_{0}^{2}+h_{c}^{2 l+2}\right\} .
\end{aligned}
$$

Finally,

$$
\begin{aligned}
\left|\left(\frac{\partial \alpha}{\partial c}\left(c_{h}\right) \frac{\partial c_{h}}{\partial t} \sigma, \sigma\right)\right| & =\left|\left(\frac{\partial \alpha}{\partial c}\left(c_{h}\right) \frac{\partial \tilde{c}}{\partial t} \sigma, \sigma\right)+\left(\frac{\partial \alpha}{\partial c}\left(c_{h}\right) \frac{\partial \xi}{\partial t} \sigma, \sigma\right)\right| \\
& \leqslant K\|\sigma\|_{0}^{2}+K \min \left(h_{p}^{-1}, h_{c}^{-1}\right)\left\|\frac{\partial \xi}{\partial t}\right\|_{0}\|\sigma\|_{0}^{2},
\end{aligned}
$$

where we pass through the use of the $L^{\infty}$-norm on either $\partial \xi / \partial t$ or $\sigma$ and then quasi-regularity to return to the $L^{2}$-norm. We are expecting to show that

$$
\|\sigma\|_{0}=O\left(h_{p}^{k+1}+h_{c}^{l+1}\right), \quad t \in J
$$

and we should like to make an induction hypothesis of the form

$$
\|\sigma\|_{0} \min \left(h_{p}^{-1}, h_{c}^{-1}\right) \rightarrow 0 \quad \text { as } h \rightarrow 0 .
$$

Let us require that

$$
\min \left(h_{p}^{k+1} h_{c}^{-1}, h_{c}^{l+1} h_{p}^{-1}\right) \rightarrow 0 \quad \text { as } h \rightarrow 0 .
$$

Note that this condition is satisfied for the natural choice of a relation between $h_{p}$ and $h_{c}$, namely that $h_{p}^{k+1}$ and $h_{c}^{l+1}$ are of the same size. Thus, if $h_{p}^{k+1}=h_{c}^{l+1}$, then

$$
h_{p}^{k+1} h_{c}^{-1}=h_{p}^{(k+1) l /(l+1)}, \quad h_{c}^{l+1} h_{p}^{-1}=h_{c}^{(l+1) k /(k+1)},
$$

and the first term always tends to zero; the second does as well if $k \geqslant 1$. If (4.18) holds, then we can expect that $\|\sigma\|_{0} \min \left(h_{p}^{-1}, h_{c}^{-1}\right)$ will tend to zero as $h$ goes to zero. 
Consequently, we assume inductively that (4.17) holds. Then,

$$
\begin{aligned}
\left|\left(\frac{\partial \alpha}{\partial c}\left(c_{h}\right) \frac{\partial c_{h}}{\partial t} \sigma, \sigma\right)\right| & \leqslant\left[\min \left(h_{p}^{-1}, h_{c}^{-1}\right)\|\sigma\|_{0}\right]^{2}\left\|\frac{\partial \xi}{\partial t}\right\|_{0}^{2}+K\|\sigma\|_{0}^{2} \\
& \leqslant \varepsilon\left\|\frac{\partial \xi}{\partial t}\right\|_{0}^{2}+K\|\sigma\|_{0}^{2}
\end{aligned}
$$

for $h$ sufficiently small.

We can sum (4.10) and (4.12) and apply the estimates (4.13), (4.14), (4.15), and (4.19) to obtain the evolution inequality

$$
\left\|\frac{\partial \pi}{\partial t}\right\|_{0}^{2}+\frac{d}{d t}\left(\alpha\left(c_{h}\right) \sigma, \sigma\right) \leqslant \delta\left\|\frac{\partial \xi}{\partial t}\right\|_{0}^{2}+K\left[\|\xi\|_{0}^{2}+\|\sigma\|_{0}^{2}+h_{c}^{2 l+2}+h_{p}^{2 k+2}\right],
$$

where, by (4.17), the constant $\delta$ can be taken as small as we like as $h$ tends to zero. The constant $K$ depends on norms of the solution of the differential system and on the value of $\varepsilon$ used in (4.15), but it does not need to depend on the choice of $\varepsilon$ used in (4.19).

We turn again to the concentration equation. We reach (3.18) as before. The term $\left(u_{h} \cdot \nabla \xi, \partial \xi / \partial t\right)$ must be treated differently in order to get to the equivalent of (3.19). Note that

$$
\begin{aligned}
\left|\left(u_{h} \cdot \nabla \xi, \frac{\partial \xi}{\partial t}\right)\right| & \leqslant\|\tilde{u}\|_{0, \infty}\|\nabla \xi\|_{0}\left\|\frac{\partial \xi}{\partial t}\right\|_{0}+\left|\left(\sigma \cdot \nabla \xi, \frac{\partial \xi}{\partial t}\right)\right| \\
& \leqslant\left[\|\tilde{u}\|_{0, \infty}+K \min \left(h_{p}^{-1}, h_{c}^{-1}\right)\|\sigma\|_{0}\right]\|\nabla \xi\|_{0}\left\|\frac{\partial \xi}{\partial t}\right\|_{0}
\end{aligned}
$$

The second term in the bracket tends to zero under the induction hypothesis (4.17), and $\|\tilde{u}\|_{0, \infty}$ can be bounded as follows. We have already assumed that $\|u\|_{0, \infty}$ is bounded; thus, it suffices to estimate $\|\rho\|_{0, \infty}$. For any $v \in V_{h}$,

$$
\begin{aligned}
\|\rho\|_{0, \infty} & \leqslant\|u-v\|_{0, \infty}+\|v-\tilde{u}\|_{0, \infty} \\
& \leqslant\|u-v\|_{0, \infty}+K h_{p}^{-1}\|u-v\|_{0}+K h_{p}^{-1}\|u-\tilde{u}\|_{0},
\end{aligned}
$$

and a choice can be made for $v$ such that

$$
\|\rho\|_{0, \infty} \leqslant K\|u\|_{k+1, \infty} h_{p}^{k} .
$$

For $k \geqslant 1,\|\tilde{u}\|_{0, \infty}$ is bounded by any number larger than the $L^{\infty}$-bound for $u$ for small $h$; for $k=0$, the bound for $\|\tilde{u}\|_{0, \infty}$ is independent of $h$, though somewhat larger than the bound for $u$ alone. In any case,

$$
\left|\left(u_{h} \cdot \nabla \xi, \frac{\partial \xi}{\partial t}\right)\right| \leqslant K\|\nabla \xi\|_{0}\left\|\frac{\partial \xi}{\partial t}\right\|_{0} \leqslant \varepsilon\left\|\frac{\partial \xi}{\partial t}\right\|_{0}^{2}+K\|\nabla \xi\|_{0}^{2},
$$

with $K$ independent of $h$ for sufficiently small $h$, provided that (4.17) holds. Thus, (3.19) remains valid, with $K_{4}$ now dependent on (4.17).

Next, note that

$$
\begin{aligned}
\left|\left(\left(u_{h}-u\right) \cdot \nabla \tilde{c}, \frac{\partial \xi}{\partial t}\right)\right| & =\left|\left((\rho+\sigma) \cdot \nabla \tilde{c}, \frac{\partial \xi}{\partial t}\right)\right| \\
& \leqslant \varepsilon\left\|\frac{\partial \xi}{\partial t}\right\|_{0}^{2}+K\left(\|\sigma\|_{0}^{2}+h_{c}^{2 l+2}\right),
\end{aligned}
$$


so that

$$
\begin{aligned}
\left(\phi \frac{\partial \xi}{\partial t}, \frac{\partial \xi}{\partial t}\right)+\frac{d}{d t} & (D \nabla \xi, \nabla \xi) \\
& \leqslant K\left(\|\xi\|_{1}^{2}+\|\sigma\|_{0}^{2}+\left\|\frac{\partial \pi}{\partial t}\right\|_{0}^{2}+h_{c}^{2 l+2}+h_{p}^{2 k+2}\right) .
\end{aligned}
$$

The inequalities (4.20) and (4.25) can be integrated in time and manipulated in the same fashion as in the previous section to obtain the following preliminary estimate:

$$
\begin{aligned}
&\|\xi\|_{L^{\infty}\left(J ; H^{1}(\Omega)\right)}+\left\|\frac{\partial \xi}{\partial t}\right\|_{L^{2}\left(J ; L^{2}(\Omega)\right)}+\|\sigma\|_{L^{\infty}\left(J ; L^{2}(\Omega)^{2}\right)} \\
&+\left\|\frac{\partial \pi}{\partial t}\right\|_{L^{2}\left(J ; L^{2}(\Omega)\right)} \leqslant K\left(h_{p}^{k+1}+h_{c}^{l+1}\right),
\end{aligned}
$$

where the constant $K$ depends on the $L^{\infty}\left(J ; W^{l+1, \infty}(\Omega)\right)$-norms of $c$ and $\partial c / \partial t$, and the $L^{\infty}\left(J ; H^{k+3}(\Omega)\right)$-norms of $p$ and $\partial p / \partial t$, and the $L^{\infty}\left(J ; W^{k+2, \infty}(\Omega)\right)$-norm of $p$. We must verify that the induction hypothesis (4.17) holds in order to finish the proof that (4.26) does hold as $h$ tends to zero.

The verification of (4.17) on the basis of the preliminary estimate (4.26) requires treating the two cases $h_{p} \leqslant h_{c}$ and $h_{p}>h_{c}$. If $h_{p} \leqslant h_{c}$, then

$$
\|\sigma\|_{0} \min \left(h_{p}^{-1}, h_{c}^{-1}\right) \leqslant K\left(h_{p}^{k+1} h_{c}^{-1}+h_{c}^{l}\right) .
$$

For $k>1$, the right-hand side of (4.27) is $O\left(h_{p}^{k}+h_{c}^{l}\right)$ and tends to zero, as desired. For $k=0$, it should be expected that $h_{p}<h_{c}$, and we must impose a relation between $h_{p}$ and $h_{c}$ to force the convergence to zero in (4.27). Let us require that there exist positive constants $\gamma_{1}$ and $\gamma_{2}$ such that

$$
\gamma_{1} \leqslant h_{p} h_{c}^{-2} \leqslant \gamma_{2} \quad \text { if } k=0
$$

then $h_{p}^{k+1} h_{c}^{-1}=O\left(h_{p}^{1 / 2}\right)=O\left(h_{c}\right)$ and (4.17) holds.

If, on the other hand, $h_{c}<h_{p}$, then

$$
\|\sigma\|_{0} \min \left(h_{p}^{-1}, h_{c}^{-1}\right) \leqslant K\left(h_{p}^{k}+h_{c}^{l+1} h_{p}^{-1}\right) \leqslant K\left(h_{p}^{k}+h_{c}^{l}\right),
$$

and, since $k \geqslant 1$, convergence to zero takes place. Thus, the inequality (4.17) holds as $h$ tends to zero, and the estimate (4.26) is fully demonstrated. Note that two constraints (4.18) for all $k$ and (4.28) for $k=0$ have been imposed.

It follows easily by integration of $\partial \pi / \partial t$ in time that

$$
\|\pi\|_{L^{\infty}\left(J ; L^{2}(\Omega)\right)} \leqslant K\left(h_{p}^{k+1}+h_{c}^{l+1}\right) .
$$

We can now combine (4.5), (4.9), (4.26), and (4.30), along with inequalities (3.4a), (3.5a), and (3.36a) of the previous section to obtain error estimates. The direct application of (4.26) and (4.30) leads to the $L^{2}$-bounds

$$
\begin{aligned}
\left\|c-c_{h}\right\|_{L^{\infty}\left(J ; L^{2}(\Omega)\right)} & +h_{c}\left\|c-c_{h}\right\|_{L^{\infty}\left(J ; H^{1}(\Omega)\right)}+\left\|\frac{\partial c}{\partial t}-\frac{\partial c_{h}}{\partial t}\right\|_{L^{2}\left(J ; L^{2}(\Omega)\right)} \\
& +\left\|p-p_{h}\right\|_{L^{\infty}\left(J ; L^{2}(\Omega)\right)}+\left\|u-u_{h}\right\|_{L^{\infty}\left(J ; L^{2}(\Omega)^{2}\right)} \\
& +\left\|\frac{\partial p}{\partial t}-\frac{\partial p_{h}}{\partial t}\right\|_{L^{2}\left(J ; L^{2}(\Omega)\right)} \leqslant K\left(h_{p}^{k+1}+h_{c}^{l+1}\right)
\end{aligned}
$$


provided that (4.18) and, if $k=0,(4.28)$ hold. It should be observed that the velocity $u$ is being approximated asymptotically to the same order as the pressure and the concentration; this should lead to better results at coarser levels of discretization than for the standard Galerkin method. Of course, more parameters are induced by the Raviart-Thomas spaces than for the usual finite element spaces for the same index $k$. An $L^{\infty}$-estimate can be given for the concentration:

$$
\left\|c-c_{h}\right\|_{L^{\infty}\left(J ; L^{\infty}(\Omega)\right)} \leqslant K\left(h_{p}^{k+1}+h_{c}^{l+1}\right) \log h_{c}^{-1},
$$

which is again almost optimal. An $L^{\infty}$-estimate for the pressure should also be derivable from an extension of the $L^{\infty}$ argument given in Johnson-Thomé [10]; since they do not offer any $L^{\infty}$ bounds for the velocity and since the velocity is still our main practical concern in the treatment of the pressure, we shall not pursue this estimate.

Department of Mathematics

University of Chicago

Chicago, Illinois 60637

I. F. BREZZI, "On the existence, uniqueness and approximation of saddle-point problems arising from Lagrangian multipliers," RAIRO Anal. Numér., v. 2, 1974, pp. 129-151.

2. Ph. G. Ciarlet, The Finite Element Method for Elliptic Problems, North-Holland, Amsterdam, 1978.

3. J. Douglas, JR., "The numerical simulation of miscible displacement," Computational Methods in Nonlinear Mechanics (J. T. Oden, ed.), North-Holland, Amsterdam, 1980.

4. J. Douglas, JR., M. F. Wheeler, B. L. Darlow \& R. P. Kendall, "Selfadaptive finite element simulation of miscible displacement in porous media," SIAM J. Sci. Statist. Comput. (To appear.)

5. J. Douglas, JR., "Simulation of miscible displacement in porous media by a modified method of characteristics procedure," Numerical Analysis, Proceedings, Dundee 1981, Lecture Notes in Math., Vol. 912, Springer-Verlag, Berlin and New York, 1982.

6. J. Douglas, JR., R. E. Ewing \& M. F. Wheeler, "The approximation of the pressure by a mixed method in the simulation of miscible displacement," RAIRO Anal. Numer., v. 17, 1983, pp. 17-33.

7. R. E. EwiNg \& M. F. WheELeR, "Galerkin methods for miscible displacement problems in porous media," SIAM J. Numer. Anal., v. 17, 1980, pp. 351-365.

8. R. E. EWING \& M. F. WheELER, "Galerkin methods for miscible displacement problems with point sources and sinks - unit mobility ratio case. (To appear.)

9. C. I. Goldstein \& R. Scotr, "Optimal $L^{\infty}$-estimates for some Galerkin methods for the Dirichlet problem," SIAM J. Numer. Anal. (To appear.)

10. C. JohNSON \& V. ThOMEE, "Error estimates for some mixed finite element methods for parabolic type problems," RAIRO Anal. Numér., v. 15, 1981, pp. 41-78.

11. J. A. Nitsche, $L_{\infty}$-Convergence of Finite Element Approximation, Proc. Second Conference on Finite Elements, Rennes, France, 1975.

12. D. W. Peaceman, Fundamentals of Numerical Reservoir Simulation, Elsevier, New York, 1977.

13. D. W. Peaceman, "Improved treatment of dispersion in numerical calculation of multidimensional miscible displacement," Soc. Pet. Eng. J., 1966, pp. 213-216.

14. P. A. Raviart \& J. M. Thomas, "A mixed finite element method for 2 nd order elliptic problems," Mathematical Aspects of the Finite Element Method, Lecture Notes in Math., Vol. 606, Springer-Verlag, Berlin and New York, 1977.

15. P. H. SAMmon, "Numerical approximations for a miscible displacement process in porous media." (To appear.)

16. A. H. Schatz, V. ThomEe \& L. B. Wahlbin, "Maximum norm stability and error estimates in parabolic finite element equations," Comm. Pure Appl. Math., v. 33, 1980, pp. 265-304.

17. R. Scorr, "Optimal $L^{\infty}$ estimates for the finite element method on irregular meshes," Math. Comp., v. 30, 1976, pp. 681-697.

18. J. M. Thomas, Sur l'Analyse Numérique des Méthodes d'Éléments Finis Hybrides et Mixtes, Thèse, Université Pierre et Marie Curie, Paris, 1977.

19. M. F. WheELER, "A priori $L_{2}$ error estimates for Galerkin approximations to parabolic partial differential equations," SIAM J. Numer. Anal., v. 10, 1973, pp. 723-759. 